\title{
The Hebrew Bible as Data: Laboratory - Sharing - Experiences
}

\author{
Dirk Roorda*,** \\ *Data Archiving and Networked Services - Royal Netherlands Academy of Arts and Sciences, \\ Anna van Saksenlaan 10; 2593 HT Den Haag, Netherlands, ${ }^{* *}$ The Language Archive - Max \\ Planck Institute for Psycholinguistics, Wundtlaan 1; 6525 XD Nijmegen, Netherlands, \\ dirk.roorda@dans.knaw.nl
}

\begin{abstract}
The systematic study of ancient texts including their production, transmission and interpretation is greatly aided by the digital methods that started taking off in the 1970s. But how is that research in turn transmitted to new generations of researchers? We tell a story of Bible and computer across the decades and then point out the current challenges: (1) finding a stable data representation for changing methods of computation; (2) sharing results in interand intra-disciplinary ways, for reproducibility and cross-fertilisation. We report recent developments in meeting these challenges. The scene is the text database of the Hebrew Bible, constructed by the Eep Talstra Centre for Bible and Computer (ETCBC), which is still growing in detail and sophistication. We show how a subtle mix of computational ingredients enable scholars to research the transmission and interpretation of the Hebrew Bible in new ways: (1) a standard data format, Linguistic Annotation Framework (LAF); (2) the methods of scientific computing, made accessible by (interactive) Python and its associated ecosystem. Additionally, we show how these efforts have culminated in the construction of a new, publicly accessible search engine SHEBANQ, where the text of the Hebrew Bible and its underlying data can be queried in a simple, yet powerful query language MQL, and where those queries can be saved and shared.
\end{abstract}

\subsection{Introduction}

The Hebrew Bible is a collection of ancient texts resulting from a ten-centuries long tradition. It is one of the most studied texts in human culture. Information processing by machines is only two centuries old, but since its inception its capabilities have evolved in an exponential manner

\section{How to cite this book chapter:}

Roorda, D. 2017. The Hebrew Bible as Data: Laboratory - Sharing - Experiences. In: Odijk, J and van Hessen, A. (eds.) CLARIN in the Low Countries, Pp. 217-229. London: Ubiquity Press. DOI: https:// doi.org/10.5334/bbi.18. License: CC-BY 4.0 
up till now (Gleick, 2011). We are interested in what happens when the Hebrew Bible as an object of study is brought under the scope of the current methods of information processing. The Eep Talstra Centre for Bible and Computing (ETCBC) formerly known as Werkgroep Informatica Vrije Universiteit (WIVU), has been involved in just this since the 1970s and their members are dedicated to this approach. The combination of a relatively stable set of data and a rapidly evolving set of methods urges for reflection. Add to that a growing set of ambitious research questions, and it becomes clear that not only reflection is needed but also action. Methods from computational linguistics and the wider digital humanities are to be used, hence people from different disciplines have to be involved. How can the ETCBC share its data and way of working productively with people that are used to a wide variety of computational ways?

In this article we tell a story of reflection and action, and the characters are databases, data formats, query languages, annotations, computer languages, archives, repositories and social media. This story has a beginning in February 2012, when a group of biblical scholars convened at the Lorentz center at Leiden for the workshop Biblical Scholarship and Humanities Computing: Data Types, Text, Language and Interpretation (Roorda et al., 2012). They searched for new ways to obtain computational tools that matched their research interests. The author was part of that meeting and had prepared a demo application: a query saver. It was an attempt to improve the sharing of knowledge. It is a craft to write successful queries for the ETCBC Hebrew Text database, and by publishing their queries, researchers might teach each other how to do it.

In the years that followed, this idea has materialised as the result of the SHEBANQ project (System for HEBrew text: ANnotations for Queries and markup), a curation and demonstrator project funded by CLARIN-NL, the Dutch department of the Common LAnguage Resource INfrastructure in Europe CLARIN. We have chosen a modern standard format for the data: Linguistic Annotation Framework (LAF), and have built a web-application for saving queries. During the execution of this project we also have built LAF-Fabric, a tool to analyze and manipulate LAF resources. At the time of writing the first version of this chapter, late 2014, we can say that we have a modern data laboratory for historico-linguistic data, plus ways to share results, not only among a small circle of theological experts, but also among computational linguists on the one hand and students and interested lay people on the other.

Of course, every beginning of such a story is arbitrary. There is always so much more that happened before. In order to provide the reader with enough context, we shall also relate key moments of that greater story. But we cannot tell the whole story: our perspective is biased to the computational side. We shall not delve into the intricacies of manuscript research, but focus on the data models and computational methods that help analyze a rather fixed body of transcribed text. Yet, we believe that this simplified context is rich enough material for a good story. Whereas this chapter deliberately scratches only the surface of the computational methods, a more technical account can be found in (Roorda et al., 2014b), which also relates how researchers have already made use of them.

\subsection{Ground Work: WIVU and ETCBC}

Since the 1970s, Eep Talstra, Constantijn Sikkel and a group of researchers at the VU University Amsterdam have been compiling a text database of the Hebrew Bible. This database started as a set of files, containing the transliterated Hebrew text of the Bible according to the Biblia Hebraica Stuttgartensia edition (Elliger and Rudolph, 1997). To this text, they added files with their observations of linguistic patterns in it as coded annotations, anchored to the individual words, phrases, clauses, and sentences. They tested tentative patterns against the data, refined them, and added manual exceptions. This led to a complex web of files, containing the base text and a set of semi-automatically generated annotations. They refrained from shaping these annotations in a hierarchical, linguistic model, because they wanted to represent observations, not theory 
(Talstra and Sikkel, 2000). The result of this work is a database in the sense of being observational data on which theories can be based. It is not a database in the sense of a modern relational database system.

The advantages of a proper (in the sense of computer science) database are obvious indeed, but the relational model does not represent textual data in a natural way, and does not facilitate queries that are linguistically meaningful. In the 1990s there have been promising efforts to define the notion of a text database. In his Ph.D. thesis, Crist-Jan Doedens (Doedens, 1994) defined a data model for texts and the notion of a topographic query language (QL) to retrieve linguistic results. He identified the relations of sequence and embedding as the key structures to store and retrieve texts. A query is topographic if its internal structure exhibits the same sequence and embedding relations as the results it is meant to retrieve. Interestingly, he did not postulate that a text is one hierarchy. In his data model, textual data may be organised by means of multiple, overlapping hierarchies.

The definition of a data model and a query language are not yet a working database system. In the 2000s, Ulrik Petersen undertook to create an implementation of Doedens's ideas. This led to the Emdros database system with the MQL (Mini-QL) query language Petersen (2004; 2006; 2002-2014). Emdros consists of a front-end, which is an MQL interpreter, and a back-end, which is an existing production class relational database system such as PostgreSQL or MySQL. Despite the fact that MQL is a concession to practicality, it is still a topographic query language and very convenient to express real-life textual queries without invoking programming skills. Since then, an Emdros export of the current Hebrew text database is being maintained by the ETCBC team. Emdros is open source software, the data model is very clear, so this export is a communication device: the intricacies of the internal annotation-creation of the ETCBC workflow are largely left behind, and users of the export have a well-defined dataset at their disposal.

\subsection{Idea: Queries As Annotations}

During the aforementioned Lorentz workshop (Roorda et al., 2012), an international group of experts reflected on how to bring biblical data resources to better fruition in the digital age. The ETCBC database had been incorporated in Bible study software, but developments there were not being driven by agendas set by academic research. Yet those bible study applications offered attractive interfaces to browse the text, look up words and more. The problem was: how can theologians, with limited ICT resources, regain control over the development of software that works with their data? The workshop offered no concrete solutions, but some ingredients of potential long-term solutions did get mentioned: open up the data and develop open source tools. Theologians can only hope to keep up with ICT developments if they allow people building on each others' accomplishments. A very concrete articulation of this statement was made by Eep Talstra himself, when he deposited the ETCBC database into EASY, the research archive of DANS (Talstra et al., 2012). It must be admitted that there remained barriers: the data was not Open Access and the format in which it was deposited was MQL, which is not a very well-known format, so the experimenting theological programmer still has a hard time to do some meaningful work with this data. But it was definitely a step towards increased sharing of resources.

In that same workshop, the author showed a demo application (Roorda, 2012) (see Figure 18.1) by which the user could browse the Hebrew text and highlight a number of linguistic features. The idea to highlight features, which are essentially annotations to the text, triggered another idea: to view queries as annotations to the passages that contain their results (Roorda and van den Heuvel, 2012). If researchers can save their carefully crafted queries as annotations, and if those annotations are centrally stored, then other researchers have access to them and may encounter them when they are reading a passage. Just as readers encounter ordinary annotations by other scholars in 


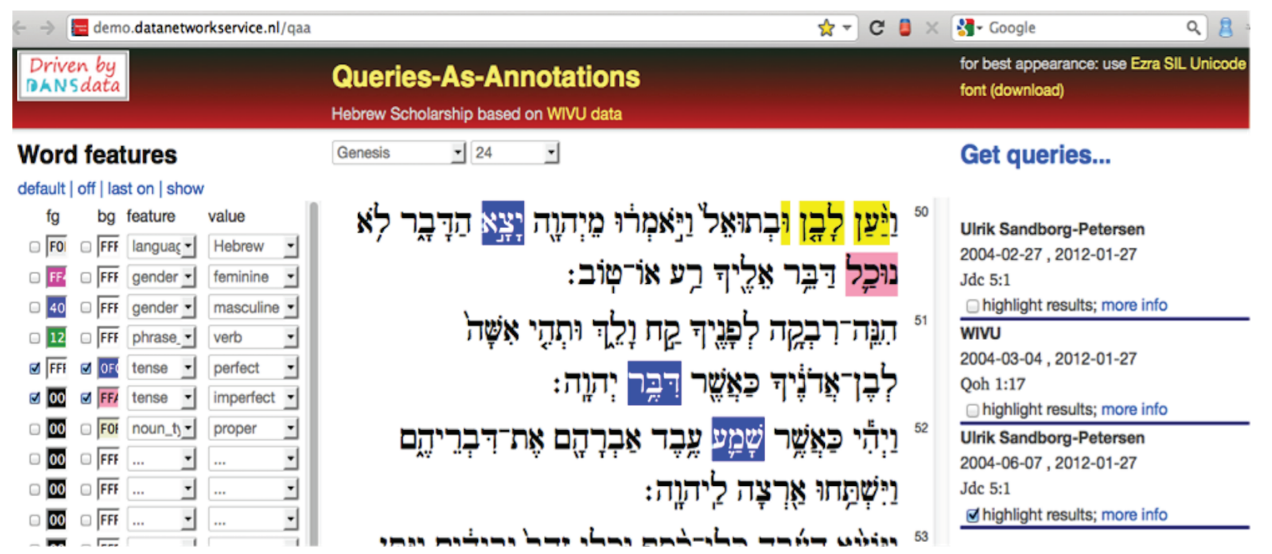

Figure 18.1: Queries/Features as Annotations.

\begin{tabular}{|l|c|c|}
\hline formalism & web app & data prep tool \\
\hline sql & 90 & 80 \\
\hline python & 250 & \\
\hline perl & & 650 \\
\hline javascript & 300 & \\
\hline html & 50 & \\
\hline css & 60 & \\
\hline shell script & & 280 \\
\hline
\end{tabular}

Table 18.1: Amount of lines of code per formalism per application.

printed books, they will encounter results of queries of others when they are browsing a chapter of the Hebrew Bible in their web browser. With a single click they are led to not only the query instruction itself but also a description of the provenance and motivation of the query. This could be the basis of interesting scenarios for cross-fertilisation.

It is interesting to note the stack of computational tools needed to write this demo. Its construction involved a data preparation tool for transforming the contents of the ETCBC database into a relational database for driving a website. The web app itself was based on Web2py, a lightweight python based web-application framework (Di Pierro, 2015).

Table 18.1 is a list of languages used to implement both the data-preparation tool and the website, together with the amount of code needed in each formalism. There are several things to note:

1. The numbers of lines of code are very small.

2. The formalisms, while considerable in number, are utterly commonplace.

3. The number of formalisms may be reduced by one by dropping Perl in favor of Python

It can be concluded that mastering commonplace ICT techniques may generate a good return on investment, in the form of a web application that expose data on the web in rich interfaces.

\subsection{Realisation: LAF-Fabric and SHEBANQ}

In 2013-2014, ETCBC together with DANS has carried out the CLARIN-NL project SHEBANQ. We seized the opportunity to implement the idea of queries-as-annotations, but to make it possible at all more work had to be done. 


\subsubsection{LAF}

First of all, a new representation of the data had to be selected, one that conformed to a standard used in linguistics. Linguistic Annotation Framework, an ISO standard (Ide and Romary, 2012), was chosen. LAF defines a data model in which an immutable stream of primary data is annotated by feature structures. The data stream is addressed by means of a graph of nodes and edges, where the nodes may be linked to regions of the primary data, and where edges serve to connect smaller parts to bigger wholes. Both nodes and edges can act as targets of annotations, which contain the feature structures. Finally, all entities, except the primary data, are serialised in XML.

In concrete terms, we have extracted the complete text of the Hebrew Bible as a plain Unicode text file. As far as LAF is concerned, this is our primary data. For the books, chapters and verses we have created nodes that are linked to the stretches of text that they correspond to. For every individual word there is a node, linked to a region defined by the character positions of the first and last character of that word. For the phrases, clauses and sentences there are nodes, linked to the regions corresponding to the words they contain. Relationships between constituents correspond to edges. The properties of sectional units, words, and constituents are key-value pairs targeted at the corresponding nodes.

For a graphic representation of how this model works, Figure 1 of (Roorda et al., 2014b) is just one tap away.

\subsubsection{LAF Versus FoLiA}

Another approach for linguistic annotations is provided by FoLiA (see chapter 6). LAF, however, is more abstract and conceptually simpler, which gave us the freedom to translate the existing data model of the ETCBC database in a straightforward way, including its more idiosyncratic data features.

\subsubsection{From EMDROS to LAF}

The LAF data model shares a lot of structure with the Emdros data model of text, objects and features. We only had to map objects to nodes and features to key-value pairs inside annotations targeting the proper nodes, so this conversion has been a straightforward process with only a few devilish details.

The result is a good example of stand-off markup. The primary data is left untouched, and around it is a graph of annotations. It is perfectly possible to add new annotations without interfering with the primary data or the other annotations. The annotations are like a fabric, into which new threads can be woven, and that can be stitched to other fabrics. In this way, the stand-off way of adding information to sources facilitates cooperation and sharing much better than adding markup inline, such as TEI prescribes. This bold assertion must be qualified by two considerations, however:

1. Stand-off markup works best in those cases where the primary sources are immutable. As easy as it is to add new annotations, so difficult it is to insert new primary data.

2. Stand-off markup flourishes in cases where the main access mode to the sources is by programmatic means. Manual inspection of stand-off data and their annotations becomes quickly overwhelming.

In our case, the first condition holds for years in a row, but updates do occur. We have dealt with that by having several copies of the database co-existing, and the website SHEBANQ exposes them all.

Table 18.2 indicates some quantities of the ETCBC data, both in their Emdros form and in their LAF form. These numbers suggest that manual inspection of individual files is so cumbersome that it pays off to invest in programmatic access of the data. 


\begin{tabular}{|l|r|r|}
\hline quantity & Emdros & LAF \\
\hline words & 426,555 & 426,555 \\
\hline linguistic objects resp. nodes & 945,726 & 945,726 \\
\hline total number of features & $22,622,100$ & $25,504,388$ \\
\hline serialised size (MQL resp. XML) & $455 \mathrm{MB}$ in 1 file & $1640 \mathrm{MB}$ in 14 files \\
\hline compiled size (SQLite3 resp. binary) & $126 \mathrm{MB}$ & $260 \mathrm{MB}$ \\
\hline
\end{tabular}

Table 18.2: Quantities in the ETCBC data.

The LAF version of the Hebrew text database has been archived at Data Archiving and Networked Services (DANS), the research archive for the humanities and social sciences in the Netherlands by Peursen and Roorda $(2014 ; 2015)$.

\subsubsection{LAF-Fabric}

As LAF is a relatively new standard, there are few LAF-compatible tools. A LAF resource is represented in XML, but the nature and size of this XML make it difficult to be handled by ordinary XML tools. Looking through the surface syntax, a LAF resource is neither a relational database, nor a document, but a graph. XML processing works well when the underlying data structure is a single hierarchy, no matter how deep, or a table of records, no matter how large, but it grinds to a halt when the data is a large and intricate web of nodes and edges, i.e. a graph.

In order to facilitate productive work with the freshly created LAF representation of the Hebrew Bible, we have developed LAF-Fabric (Roorda, 2013-2014b), which is a LAF compiler and loader. In a typical workflow, a researcher wants to inspect the LAF data, focus on some aspects, sort, collate, link and transform selected data, and finally export results. Without LAF-Fabric, the obvious way to do so is read the XML data, apply XPATH, XSLT or XQUERY scripts and collect the results. Reading the XML data means parsing it and building an internal representation in memory, and this alone takes an annoying 15 minutes on a average laptop and uses a prohibitive amount of memory. This is not conducive to an interactive, explorative, agile use of the data, and LAF-Fabric remedies this. When first invoked on a LAF-resource, it compiles it into efficient data structures and writes those to disk, in such a way that this data can be loaded fast. This one-time compilation process takes roughly 15 minutes, but then the data loads in a matter of seconds every time you want to work with it. Furthermore, LAF-Fabric offers a programmers interface (API) to the LAF data, by which the programmer can walk over the nodes and edges and collect feature information on the fly. These walks are fast, and can be programmed easily.

The idea to create LAF-Fabric arose after we tried to use a library called graf-python (Bouda, 2013-2014), part of POIO (Bouda et al., 2012), for the biblical LAF data. Unfortunately, the way graf-python was programmed made it unsuitable for dealing with our LAF resource because of its size. Python is a scripting language with a clean syntax and a good performance if used judiciously, hence we undertook to write LAF-Fabric in Python as well. We use those parts of Python that perform best for the heavy data lifting, and those parts that are most user friendly for the programmers interface. LAF-Fabric is a package that can be imported in any Python script, and it behaves particularly well when invoked in an IPython notebook. It is available on the Python Package Index (Roorda, 2013-2014b), so that a user can get it by just typing on the command line.

pip3 install laf-fabric

IPython Notebook is an interactive way of writing Python scripts and documentation (Pérez and Granger, 2007). A notebook is a document in which the data analyst writes cells with Python code and other cells with documentation. Code cells can be run individually, in any order, while the results of the execution remain in memory. The notebook has powerful capabilities of formatting 

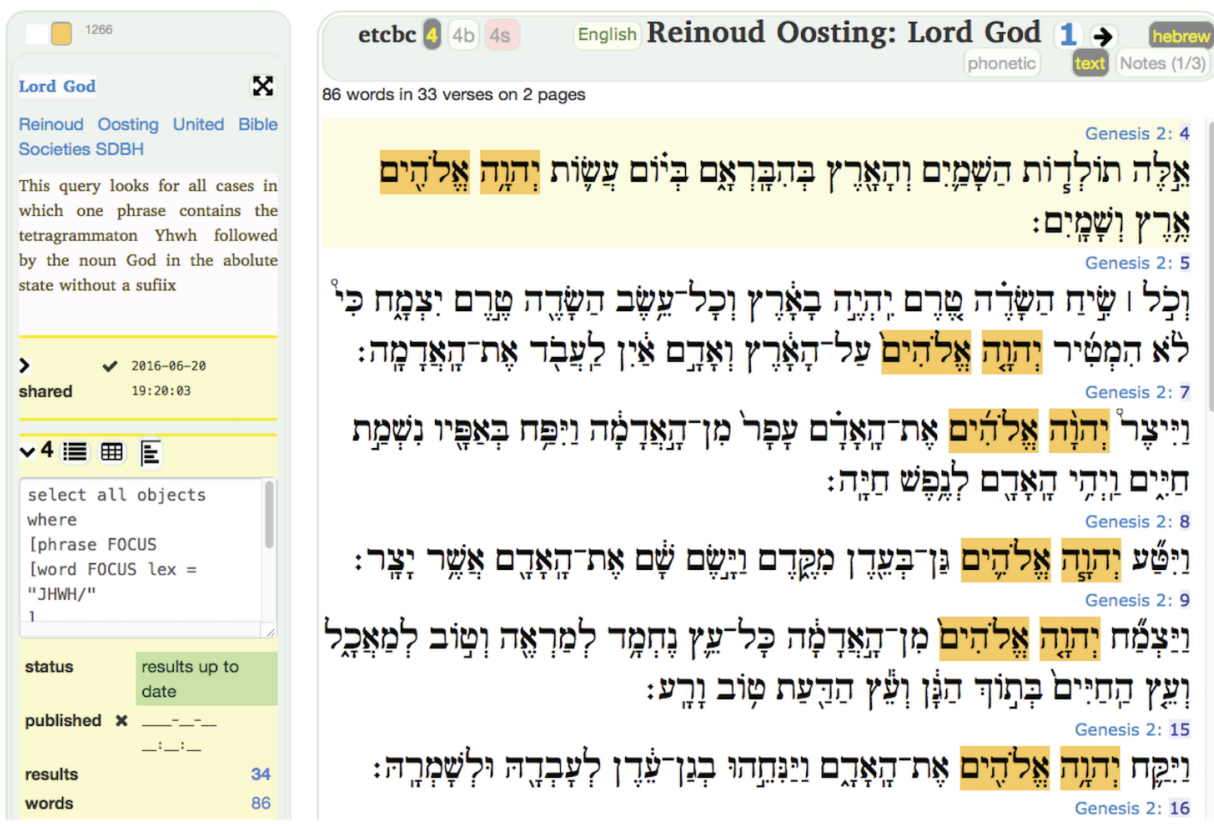

Figure 18.2: A saved query in SHEBANQ.

results. A notebook can be published easily on the web, so that others can download it and execute it as well, provided they have the same data and packages installed. IPython notebook belongs to a branch of computer programming called scientific computing. It is about explorative data analysis by means of computing power. The scientific programmer produces analyses, charts, and documents that account for his data and results. By contrast, the typical software engineer produces applications that perform well-defined tasks for end users. The scientific programmer works close to the researchers, and writes special purpose code fast, and reacts to changing demands in an agile way. The software engineer works at a greater distance from the actual use cases. He uses programming languages that support good software organisation at the cost of a much slower development process. He is less prepared to accomodate fast-changing requirements.

When LAF-Fabric runs in an IPython notebook, even the few seconds it needs to load data are required only once. The programmer can experiment with his code cells at will, without the need to reload the data all the time.

LAF-Fabric has already been used for some significant data extractions. There is a varied and growing set of notebooks (Roorda, 2014a) on Github that is testimony to the extent of use cases that can be served. Not only data analysis, but also adding new annotations is supported. One of the use cases is the query saver itself.

\subsubsection{Text-Fabric}

From the start of 2017 onwards, I have deprecated LAF-Fabric in favour of a new format and tool: Text-Fabric. The data fits now easily in a Github repository: BHSA.

\subsubsection{SHEBANQ: Demonstrator}

The actual goal of the SHEBANQ project was to create a demonstrator query saver for the ETCBC data. This has been achieved, and the resulting web application is called SHEBANQ (van Peursen et al., 2014). 
It went live on 2014-08-01, and contains now, on 2016-06-24, 609 shared queries, saved by 84 users of a total of 293 registered users. The public part of the application offers users the options to read the Hebrew Bible chapter by chapter, to see query results of public queries as annotations in the margin, and to jump from query annotations to query descriptions and result lists. Figure 18.2 shows a screenshot of the page of a saved query.

When a user clicks on the passage indicator of a result, he is led to the relevant chapter, where other queries show up and can be navigated to, see Figure 18.3.

When users register and $\log$ in, they can write their own queries, have them executed, save them, including the query results, and make them public.

\subsubsection{SHEBANQ: Research Environment}

If the demonstrator shows one thing, then it is the fact that there are many additional desiderata. Whereas SHEBANQ has been designed to fulfill the sharing function, researchers also want to use it as a research tool. It is not easy to write a good MQL query, because many of the linguistic aspects of the data are not shown on the interface. If, for instance, a user wants to use the dictionary entries of the words or the syntactic features of clauses and phrases, he has no immediate, visual clues. So we added linguistic information in layers below the plain text, accessible by a tap on the verse number, see Figure 18.4.

Other additions we have made are:

- easy access to lexical information by clicking on words;

- clickable charts for query results and word occurrences, Figure 18.5);

- CSV exports of data;

- support for multiple versions of the data;

- manual annotation, also with images; bulk-uploadable annotations, e.g. cross-references;

- corpus-driven phonetic transcription;

- user manual, query manual, and feature documentation one tap away;

- generation of citable links of page views, and published queries.

One particular addition is a consequence of the success of SHEBANQ: more and more users leave their queries in SHEBANQ, and the need is felt to suppress queries from the margin. For this muting has been added.
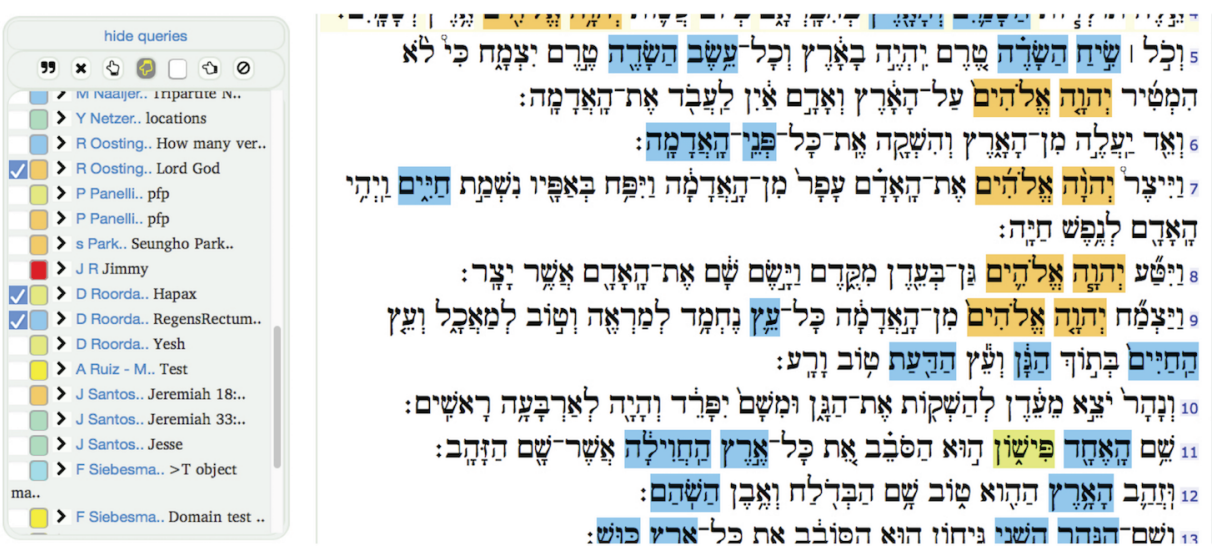

Figure 18.3: Reading a passage and seeing the results of various queries. 


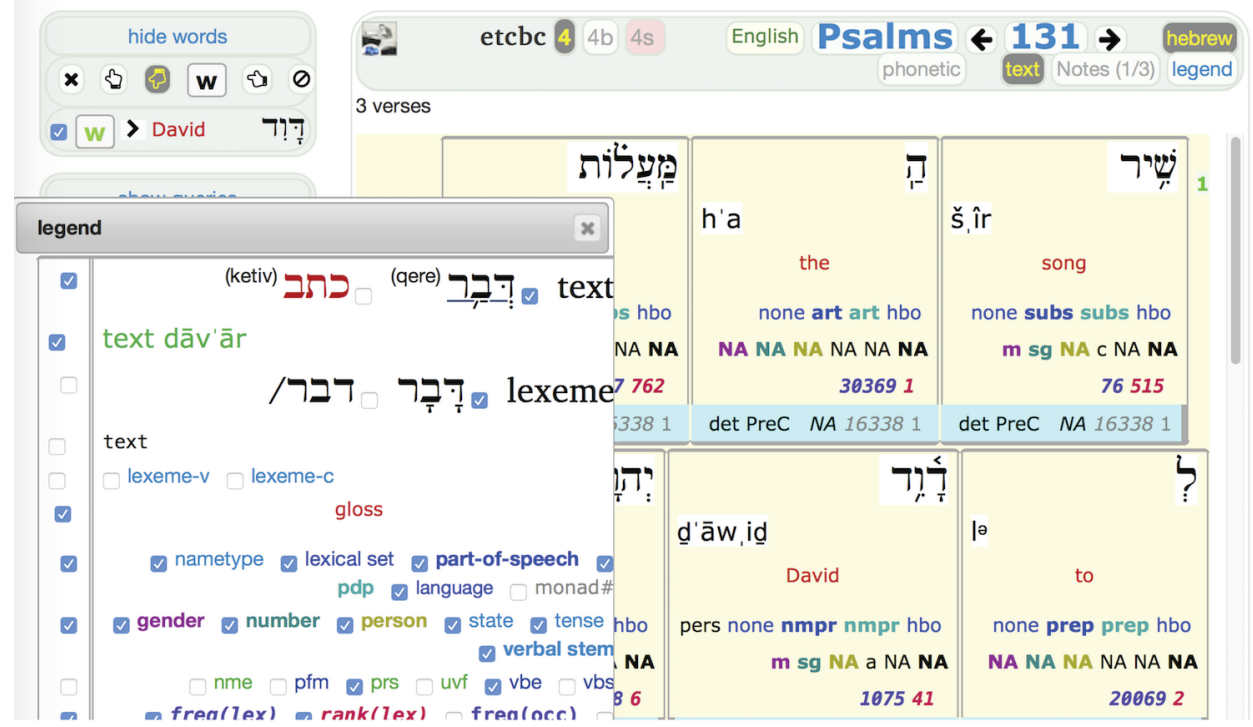

Figure 18.4: Text and underlying data.
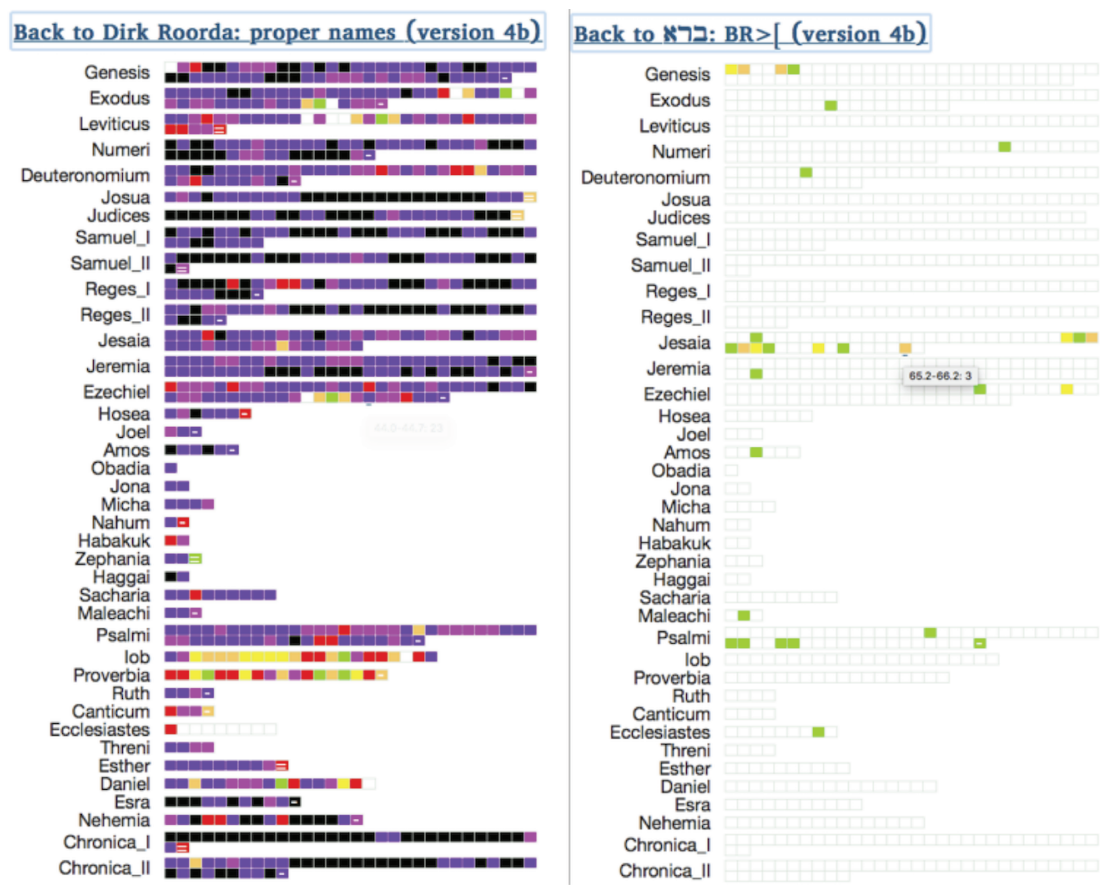

Figure 18.5: Clickable charts; left: proper name occurrences; right: the verb create.

\subsection{Reflection}

Back in 2012 we faced the challenge to provide better data models and better programs for biblical scholars. It had become clear that the software companies that were developing the bible study applications were not interested in building software for researchers. The researchers did not have 
access to programmers' time. There seemed to be only one way out: researchers should take their fate in their own hands and write the software themselves, which looked like a daunting proposition at best and an impossible one at worst. Yet, in 2014, we have created a publicly accessible tool for querying the linguistic data of the Hebrew Bible, with a means to share those queries. We also have a data laboratory where the programming theologian can take control over her data. Collectively, biblical scholars can use the data laboratory to help the query tool evolve according to their needs. Several factors have contributed to this achievement.

1. The existence of the LAF standard, which turned out to be a natural fit for this kind of data.

2. The realisation that the plain text of the Hebrew Bible is not subject to copyright, and hence that the ETCBC database of text and annotations can be made available as Open Source.

3. The existence of a research archive, DANS, acting as a data-hub; the intellectual heritage of many years of ETCBC work lays deposited there and is open to scrutiny by anyone at any time.

4. The existence of a social medium for program code, Github; all software for LAF-Fabric and SHEBANQ (and even some of the supporting software) lies there ready to be cloned and re-used.

5. The rise of scientific computing and its paraphernalia, such as (interactive) Python and auxiliary packages; it offers an unprecedented level of user-friendliness to novice programmers; it has the potential to draw a much wider range of humanities scholars into the enticing world of computing. A researcher is much closer to a scientific programmer than to a software engineer.

Yet, this is not sufficient to get the job done. The ETCBC is steeped in its own ways, it has an efficient internal data workflow, run with the best tools that were available in the late 1980s. The internet existed then, but had not yet morphed into the world-wide web. Data sharing is not in the genes of the ETCBC. Doing unique things in relative isolation for a prolonged stretch of time tends to make you idiosyncratic. The ETCBC has its own transliteration of Hebrew, its own, locally documented way of coding data into forms that are optimal for local data processing.

Opening up to the world poses new requirements on the ways the data is coded and how it is documented. Along with archiving the existing ETCBC documentation at DANS, we have published a new kind of feature documentation on the web (Roorda et al., 2014a). There we document not only the intended meaning of features, but we also provide frequency lists of their complete value sets, things that are easily computed by means of LAF-Fabric.

Can we say that we have succeeded in meeting the challenges posed in 2012? The big question is: will there be enough programming theologians to make this a viable route? Proof of success would be the adoption of LAF-Fabric by at least some theological researchers, interest in the Hebrew data from the side of computational linguistics and artificial intelligence.

Here are some indicators:

- there is a small but growing group of LAF-Fabric users: programming theologians;

- works using LAF-Fabric and/or citing queries in SHEBANQ: (Roorda et al., 2014b), Kalkman 2013; 2015, (de Vree, 2016);

- several courses on MQL queries have been given, in the US and the Netherlands;

- SHEBANQ interlinks with Bible Online Learner (Winther-Nielsen and Tøndering, 2013).

Because all these activities occur in the open, people interested in these techniques find each other through Github, Academia.edu, Twitter, and keep in touch through media such as Slack. Time will tell if the pull exerted by the digital methods will be enough to keep this nascent community together. 


\section{Acknowledgements}

The following people contributed significantly to the work described in this chapter, in very different ways:

Ulrik Petersen for creating EMDROS, providing it as Open Source software and maintaining it all those years.

The ETCBC people: Eep Talstra and Constantijn Sikkel for creating and maintaining the ETCBC database and sharing so much knowledge about it; Wido van Peursen for paving the way for increased sharing of data. Oliver Glanz (now Andrews University Michigan) for showing order in the forest of features of the database; Gino Kalkman and Martijn Naaijer for using the new tools and challenging me. Grietje en Johan Commelin for their efforts to get LAF-Fabric working in the cloud. Reinoud Oosting for finding subtle bugs in SHEBANQ and Janet Dyk introducing new use cases.

Wider Digital Humanities: Joris van Zundert (HuygensING) for leading an inspiring Interedition bootcamp (van Zundert, 2012) which set me on the track of rapid development for the humanities; Rens Bod (Univ. of Amsterdam) and Andreas van Cranenburgh (Univ. of Amsterdam and HuygensING) who asked for the Hebrew data as tree structures in order to try out Data Oriented Parsing for classical Hebrew.

Last but not least: my colleagues at DANS: Henk Harmsen and Andrea Scharnhorst for granting additional time for research in these topics; Henk van den Berg and Heleen van de Schraaf for developing fundamental portions of the web-service and web application of SHEBANQ.

\section{References}

Bouda, Peter (2013-2014), graf-python. Python software on Github. https://gi thub.com/cidles/ graf-python.

Bouda, Peter, Vera Ferreira, and António Lopes (2012), Poio API - an annotation framework to bridge language documentation and natural language processing., Proceedings of The Second Workshop on Annotation of Corpora for Research in the Humanities, Lisbon, 2012, Lisbon, Portugal. ISBN: 978-989-689-273-9, http://alfclul.clul.ul.pt/crpc/acrh2/ACRH-2_papers/ Bouda-Ferreira-Lopes.pdf.

de Vree, Frederik (2016), Using social co-occurrencenetworks to analyze biblicalnarrative. Master Thesis, VU Amsterdam. https://github.com/Fred-Erik/social-biblical-networks, https://www. academia.edu/26149493/Using_social_co-occurrence_networks_to_analyze_ Biblical_narrative.

Di Pierro, Massimo (2015), Web2py. full stack web framework, 6th edition. Online book. http: //web2py.com/book.

Doedens, Crist-Jan (1994), Text Databases. One Database Model and Several Retrieval Languages, number 14 in Language and Computers, Editions Rodopi, Amsterdam, Netherlands and Atlanta, USA. ISBN: 90-5183-729-1, http: //books. google. nl/books?id=9gg0BRz1d04C.

Elliger, Karl and Wilhelm Rudolf Rudolph, editors (1997), Biblia Hebraica Stuttgartensia, 5th corrected ed., Deutsche Bibelgesellschaft, Stuttgart, Germany. http: //www. bibelwi ssenschaft. de/ startseite/wissenschaftliche-bibelausgaben/biblia-hebraica/bhs/.

Gleick, James, editor (2011), The Information: a History, a Theory, a Flood, HarperCollins. ISBN: 0007225741, http://en.wikipedia.org/wiki/The_Information:_A_History,_a_Theory, _a_Flood.

Ide, Nancy and Laurent Romary (2012), Linguistic Annotation Framework. ISO standard 24612:2012. Edition 1, 2012-06-15. http://www. iso.org/iso/home/store/catalogue_tc/ catalogue_detail.htm?csnumber $=37326$. 
Kalkman, Gino J. (2013), Functions of asyndetic clause relations in biblical Hebrew. IPython Notebook. http://nbviewer. ipython.org/gi thub/ETCBC/Biblical_Hebrew_Analysis/blob/master/ Miscellaneous/AsyndeticClauseFunctions.ipynb.

Kalkman, Gino J. (2015), Verbal Forms in Biblical Hebrew Poetry: Poetical Freedom or Linguistic System?, PhD thesis, VU University, Amsterdam. https://shebanq. ancient-data.org/tools? goto=verbsystem.

Pérez, Fernando and Brian E. Granger (2007), IPython: a system for interactive scientific computing, Computing in Science and Engineering 9 (3), pp. 21-29, IEEE Computer Society. http: //ipython.org, ISSN: 1521-9615, DOI: http://dx.doi.org/10.1109/MCSE.2007.53.

Petersen, Ulrik (2002-2014), Emdros. text database engine for analyzed or annotated text. Open Source software. http://emdros.org.

Petersen, Ulrik (2004), Emdros - a text database engine for analyzed or annotated text, Proceedings of COLING 2004, p. 1190-1193. http://emdros .org/petersen-emdros-COLING-2004 . pdf.

Petersen, Ulrik (2006), Principles, Implementation Strategies, and Evaluation of a Corpus Query System, Vol. 4002, Springer, p. 215-226. http://link. springer.com/chapter/10.1007\% 2F11780885_21.

Peursen, Wido Th. and Dirk Roorda (2014), Hebrew text database ETCBC4. Dataset available online at Data Archiving and Networked services, Den Haag, Netherlands. DOI: http://dx. doi.org/10.17026/dans-2z3-arxf.

Peursen, Wido Th. and Dirk Roorda (2015), Hebrew text database ETCBC4b. Dataset available online at Data Archiving and Networked services, Den Haag, Netherlands. DOI: http://dx. doi.org/10.17026/dans-z6y-skyh.

Roorda, Dirk (2012), Queries-as-annotations. Github repository. https://gi thub.com/Dans-labs/ annotation-paradigm.

Roorda, Dirk (2013-2014b), LAF-Fabric. workbench for analysing LAF resources. Python software on the Python Package Index with source on Github. https://github.com/Dans-labs/ laf-fabric, https://pypi.python.org/pypi/laf-fabric.

Roorda, Dirk (2014a), LAF-Fabric notebooks. examples of data processing for the hebrew bible. Python software on Github. https://shebanq. ancient-data.org/tools, https://gi thub.com/ ETCBC/laf-fabric-nbs.

Roorda, Dirk and Charles M.J.M. van den Heuvel (2012), Annotation as a new paradigm in research archiving, Proceedings of ASIS\& 2012 Annual Meeting. Final Papers, Panels and Posters. https://www. asis.org/asist2012/proceedings/Submissions/84.pdf (author's version: http: //arxiv.org/abs/1412.6069).

Roorda, Dirk, Constantijn Sikkel, and Wido Th van Peursen (2014a), Feature documentation of shebanq. Github repository published on readthedocs. https://etcbc.gi thub.io/bhsa/.

Roorda, Dirk, Gino Kalkman, Martijn Naaijer, and Andreas van Cranenburgh (2014b), Laf-fabric: a data analysis tool for linguistic annotation framework with an application to the hebrew bible, Computational Linguistics in the Netherlands Journal 4, pp. 105-120, CLIN. http: //www. clinjournal.org/sites/clinjournal.org/files/08-Roorda-etal-CLIN2014.pdf.

Roorda, Dirk, Jan Krans, Bert-Jan Lietaert-Peerbolte, Wido Th. van Peursen, Ulrik SandborgPetersen, and Eep Talstra (2012), Scientific report of the workshop biblical scholarship and humanities computing: Data types, text, language and interpretation, held at the lorentz centre leiden from 6 feb 2012 through 10 feb 2012, Technical report, Lorentz Center, Leiden. http://www. lorentzcenter.nl/1c/web/2012/480/report. php3?wsid=480\& venue $=$ Oort.

Talstra, Eep and Constantijn J. Sikkel (2000), Genese und kategorienentwicklung der wivudatenbank, in Hardmeier, C. et al, editor, Ad Fontes! Quellen erfassen - lesen - deuten. Wat ist Computerphilologie? Ansatzpunkte und Methodologie - Instrument und Praxis, VU University Press, Amsterdam, Netherlands, pp. 33-68. 
Talstra, Eep, Constantijn J. Sikkel, Oliver Glanz, Reinoud Oosting, and Janet W. Dyk (2012), Text database of the hebrew bible. Dataset available online after permission of the depositor at Data Archiving and Networked services, Den Haag, Netherlands. DOI: http://dx.doi.org/ $10.17026 /$ dans $-x 8 h-y 2 b v$.

van Peursen, Wido Th., Dirk Roorda, Henk van den Berg, and Heleen van de Schraaf (2014), SHEBANQ, a search engine and query saver for the etcbc text database of the hebrew bible. web application. https://shebanq. ancient-data.org.

van Zundert, Joris et al. (2012), Proceedings of the leuven2012 interedition workshop. Wiki page. http://interedition.eu/wiki/index.php/Leuven2012_Proceedings.

Winther-Nielsen, Nicolai and Claus Tøndering (2013), Bible online learner. Website, learning system for Hebrew students. http://bibleol. 3bmoodle.dk/. 